\section{MEANCOMP: A Pascal program for computing mean reaction times with outlier selection}

\author{
PAUL WHITNEY \\ University of Arkansas at Little Rock \\ Little Rock, Arkansas
}

Reaction time has become a ubiquitous dependent measure in cognitive psychology. The MEANCOMP program was written primarily to compute mean reaction times (RTs) for each subject participating in several conditions of an experiment. Typically, a subject is given a number of trials in each condition. The mean RTs for each subject in each condition are then used in the statistical analyses of the experiment. However, the distributions of some of the subject's original RTs may contain outliers at the upper end, and these scores can distort the results. In most research using RTs, the outliers are identified by one of several methods and are discarded because they are outside the time course reasonably expected for the execution of the processes under investigation. Such scores are usually considered to be errors resulting from a momentary distraction or lapse of attention, and they do not enter into the calculation of the subject's RTs. It is unusual for more than a small percentage of the total scores to be discarded.

\section{Overview}

MEANCOMP is an interactive Pascal program that allows the user to choose from among three methods of outlier selection. The user may also choose to ignore outliers. After each subject's data are entered, outliers (if any) and the mean RTs for each condition are shown. Missing data and the number of outliers are counted. The program also creates a file that contains the mean RTs in a format suitable to be used to build a source file for one of the major statistical packages (e.g., BMDP, SAS, SPSS).

\section{Input}

Outlier Selection. If the user decides to identify outliers, any one of three options can be employed. Scores can be discarded because they exceed (1) a user-supplied cutoff score, (2) a critical value equal to a specified number of standard deviations above the subject's overall mean RT, or (3) a critical value determined by the Dixon ratio of ranges test (Dixon, 1950, 1951). The former two methods are frequently reported in the current cognitive literature. The Dixon test has been used on occasion (e.g.,

I would like to express my appreciation to Ed Wike for his suggestions concerning the outlier options included in this program. Preparation of this article was supported by the Marie Wilson Howell's bequest to the Psychology Department, University of Arkansas at Little Rock. The author's mailing address is: Department of Psychology, University of Arkansas at Little Rock, 33rd and University, Little Rock, AR 72204.
Ashcraft, 1978) and has the advantage of being more thoroughly investigated by statisticians interested in outliers (see Barnett \& Lewis, 1978; Wike, 1971).

Data Entry. The user supplies the number of conditions and the number of scores per condition. In its current version, MEANCOMP will accept up to 200 RTs per subject. This maximum can be altered easily to fit the user's needs. The program asks the user for a subject identifier (up to six characters) and then accepts the subject's data typed by condition. The scores for each condition can be typed on any number of lines desired. Missing data, such as scores lost due to mechanical failure, are coded as a string of four zeros. The rationale for this method of coding was that the user will typically be entering three- or four-digit RTs, and zero will never be an actual score.

\section{Output}

When the program has accepted a given subject's last score, outliers, if any, are shown along with the mean RTs for each condition. When all the subjects have been processed and the program is terminated, the total number of scores entered is displayed, as are the counts of outliers and missing data. The data file that is created by MEANCOMP is called "outdata," and it contains the mean RTs for each subject in each condition. Each subject's means occupy one line of the file, with the means separated by a single space.

\section{Hardware}

The program is written in standard Pascal (Jensen \& Wirth, 1974) and implemented on a Vax 11 using the Vax/VMS (version 4.2) operating system. The only modifications that should be necessary for implementation on other systems are in the commands for opening and writing to the "outdata" file, because these commands differ slightly from system to system.

\section{Availability}

A listing of the program, complete with documentation, is available from the author at no cost.

\section{REFERENCES}

AshCraft, M. H. (1978). Property dominance and typicality effects in property statement verification. Journal of Verbal Leaming \& Verbal Behavior, 17, 155-164.

BARNETt, V., \& LeWIS, T. (1978). Outliers in statistical data. Chichester: Wiley.

Dixon, W. J. (1950). Analysis of extreme values. Annals of Mathematical Statistics, 21, 488-506.

Dixon, W. J. (1951). Ratios involving extreme values. Annals of Mathematical Statistics, 22, 68-78.

Jensen, K., \& WiRTH, N. (1974). Pascal user manual and report (2nd ed.). New York: Springer-Verlag.

WIKE, E. L. (1971). Data analysis: A statistical primer for psychology students. Chicago: Aldine Atherton.

(Revision accepted for publication January 29, 1986.) 